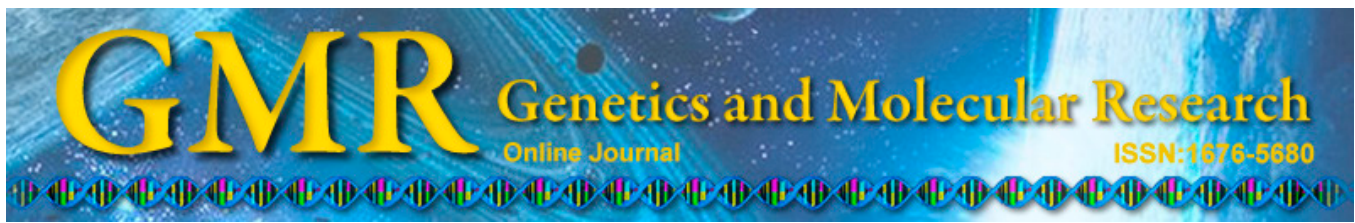

\title{
Cloning and sequence analysis of the Blumea balsamifera DC farnesyl diphosphate synthase gene
}

\author{
Y.X. Pang, L.L. Guan, L.F. Wu, Z.X. Chen, K. Wang, X.L. Xie, F.L. Yu, \\ X.L. Chen, Y.B. Zhang and Q. Jiang \\ Tropical Crops Genetic Resources Institute, \\ Chinese Academy of Tropical Agricultural Sciences, Haikou, China \\ Corresponding author: Y.X. Pang \\ E-mail: blumeachina@126.com
}

Genet. Mol. Res. 13 (4): 9874-9882 (2014)

Received April 2, 2014

Accepted August 13, 2014

Published November 27, 2014

DOI http://dx.doi.org/10.4238/2014.November.27.15

\begin{abstract}
Blumea balsamifera DC is a member of the Compositae family and is frequently used as traditional Chinese medicine. Blumea balsamifera is rich in monoterpenes, which possess a variety of pharmacological activities, such as antioxidant, anti-bacteria, and antiviral activities. Farnesyl diphosphate synthase (FPS) is a key enzyme in the biosynthetic pathway of terpenes, playing an important regulatory role in plant growth, such as resistance and secondary metabolism. Based on the conserved oligo amino acid residues of published FPS genes from other higher plant species, a cDNA sequence, designated $B b F P S$, was isolated from $B$. balsamifera DC using polymerase chain reaction. The clones were an average of $1.6 \mathrm{~kb}$ and contained an open reading frame that predicted a polypeptide of 342 amino acids with $89.07 \%$ identity to FPS from other plants. The deduced amino acid sequence was dominated by hydrophobic regions and contained 2 highly conserved DDxxD motifs that are essential for proper functioning of FPS. Phylogenetic analysis indicated that FPS grouped with other
\end{abstract}


composite families. Prediction of secondary structure and subcellular localization suggested that alpha helices made up $70 \%$ of the amino acids of the sequence.

Key words: Blumea balsamifera DC; Farnesyl diphosphate synthase; Phylogenetic analysis; Secondary structure; Subcellular localization

\section{INTRODUCTION}

Blumea balsamifera DC is one of the most important medicinal plants in China and is chiefly distributed in southern China. It has long been used as a tea and as a cure for various disorders such as rheumatism and hypertension. The leaves possess various physiological activities, including plasmin-inhibitory, antifungal, and liver-protective effects (Norikura et al., 2008; Saewan et al., 2011). An infusion of the leaves is used as a stomachic, carminative, diaphoretic, expectorant, and emmenagogue. A decoction from fresh leaves alone or in combination with other plants is used as a bath for women after giving birth (Ruangrungsi et al., 1985). Several studies on the chemical constituents of B. balsamifera DC have been reported and a number of flavanoids have been isolated from this plant. The essential oil of $B$. balsamifera contains 1-borneol, cineole, limonene, and palmitic and myristic acid, sesquiterpene alcohol, dimethyl ether, pyrocatechic tannin, glycoside, and levorotatory camphor (Ruangrungsi et al., 1981; Barua and Sharma, 1992; Fazilatun et al., 2001, 2004; Ali et al., 2005). Additionally, the flavonoids present in B. balsamifera DC leaves extracts are potent antioxidants (Fazilatun et al., 2004).

Farnesyl diphosphate synthase (FPS) is a key enzyme in the biosynthetic pathway of terpenes, playing an important regulatory role in plant growth, such as resistance and secondary metabolism. It catalyzes 2 consecutive condensations of isopentenyl diphosphate with allylic diphosphates, dimethylallyl diphosphate, and the resulting geranyl diphosphate into FPP, which is used in the biosynthesis of sterols, dolichols, mitochondrial electron transfer chain components, prenylated proteins, and a wide range of sesquiterpenoids including phytoalexins. Genes encoding FPPS have been cloned from many species, including rat (Clarke et al., 1987), human (Sheares et al., 1989), Saccharomyces cerevisiae (Anderson et al., 1989), Arabidopsis thaliana (Delourme et al., 1994), and Lupinus albus (Attucci et al., 1995).

In this study, based on similarity of cDNA sequences, homologous primers were designed according to the highly conserved region of Farnesyl diphosphate synthase (FPS) genes of other species, and then the full-length cDNA sequence of FPS was isolated from B. balsamifera DC using reverse transcription-polymerase chain reaction (RT-PCR) and rapid amplification of cDNA ends (RACE). Sequence analyses were conducted using the DNAMAN, DNAStar, Mega, and BLAST (http://blast.be-md.ncbi.nlm.nih.gov/Blast.cgi) software.

\section{MATERIAL AND METHODS}

\section{Plant materials}

B. balsamifera DC was grown in a natural environment at the experimental fields of Tropical Crops Genetic Resources Institute. Young leaves were harvested, snap-frozen in liquid nitrogen, and stored at $-80^{\circ} \mathrm{C}$ until RNA extraction. 


\section{RNA extraction and $R T$ reactions}

Total RNA was isolated from young leaves using the Plant RNA Extraction Kit (Tiangen, Beijing, China). RNA concentration was determined spectrophotometrically, and verified by ethidium bromide staining on an agarose gel. Total RNA was then treated with RNase-free DNaseI (TaKaRa, Shiga, Japan) to remove any contaminating genomic DNA, and approximately $3 \mu \mathrm{g}$ RNA was used as a template for the first cDNA synthesis using TaKaRa reverse transcription reagents following manufacturer instructions; samples were stored at $-20^{\circ} \mathrm{C}$.

\section{Isolation of partial cDNA clones}

Clones were amplified using the cDNA described above as a template and PF1/PR1 primers designed from the FPS sequences conserved in other plant species. The primer sequences were as follows: PF1: 5'-CTTGGTTGGTGCATTGAATGG-3' and PR1: 5'-TAAAAT GAGT AGTAAGCAGT TTTG-3'. PCR was performed as follows: $95^{\circ} \mathrm{C}$ for $5 \mathrm{~min}$, followed by 35 cycles at $94^{\circ} \mathrm{C}$ for $30 \mathrm{~s}, 42^{\circ} \mathrm{C}$ for $30 \mathrm{~s}$, and $72^{\circ} \mathrm{C}$ for $1 \mathrm{~min}$, then $72^{\circ} \mathrm{C}$ for $7 \mathrm{~min}$. One DNA fragment with the same expected size was generated, subcloned into the vector pMD19-T (Promega, Madison, WI, USA), and sequenced.

\section{RACE}

The 5'-ends of FPS cDNAs were amplified using the SMART and RACE methods with the 5'-RACE System (BD-Clontech, Palo Alto, CA, USA) according to manufacturer instructions with 2 different antisense primers: PR2 (CGGCGATGAATAGACAATG) and PR3 (CCAGAGGCTGTTTGGAACT), which were deduced from the 5'-region specific to each of the clones described above (Alam et al., 2010). Amplification of the 3'-end was performed using the 3'-RACE method as described by Schmidt et al. (2010). The primers used for 3'-RACE were the oligo (dT) 17 adaptor primer (for first-strand cDNA synthesis) and the forward primer PF2 (CCCTGTTGGTTTAGATTACCC) according to manufacturer instructions.

Based on the sequence information of the 5'- and 3'-ends, the sense/antisense primer pairs PF4 (ATGAGCAGCGATCTGAAGT) and PR6 (CTACTTTTGCCTCTTGTATA TCTTTC) were designed to amplify the open reading frame of FPS. PCR fragments were subcloned into the vector pMD19 -T (Promega) and transformed into Escherichia coli JM109 cells. The nucleotide sequence was determined by Invitrogen (Carlsbad, CA, USA).

\section{Sequence analyses}

The nucleotide sequence from our cDNA clone and the deduced amino acid (aa) sequence were identified using the National Center for Biotechnology Information BLAST program (http://www.ncbi.nlm.nih.gov/BLAST/). Sequence alignment was conducted using the DNAMAN software (version 4.0, Lynnon Biosoft, Quebec, Canada). A phylogenetic tree was constructed using the neighbor-joining algorithm, implemented in the MEGA4.0 software (Tamura et al., 2007). The cDNA sequence and deduced aa sequence were designated as BbFPS. 


\section{RESULTS AND DISCUSSION}

\section{Nucleotide sequence analysis}

Using the degenerate primers PF1/PR1, a distinct cDNA fragment was amplified with an expected size of approximately 600 base pairs (bp), containing an open reading frame encoding a 200-aa protein. Alignment of the deduced aa sequences showed a high degree of identity between our cloned fragment and the central coding regions of known plant FPS sequences. The fragment was then used to design gene-specific primers to clone the full-length FPS sequence. A 500-bp fragment of 5' RACE and a 900-bp fragment of 3' RACE were amplified; both sequences were determined. BLAST searches of the deduced aa sequences revealed correspondence with known FPS genes. Sequence comparisons of the $5^{\prime}$ and $3^{\prime}$ ends with the central region of the gene indicated a perfect match between overlapping regions, suggesting that these sequences represent the missing regions of FPS genes. Based on this sequence data, specific primers were designed and the full-length cDNAs were amplified, cloned, and sequenced, revealing $100 \%$ identities to the expected FPS sequence. Our sequence analysis indicated that the full-length cloned cDNA (1597 bp) contained a 1029-bp open reading frame encoding 342 aa residues with an estimated molecular mass of $39.188 \mathrm{kDa}$ (Figure 1).

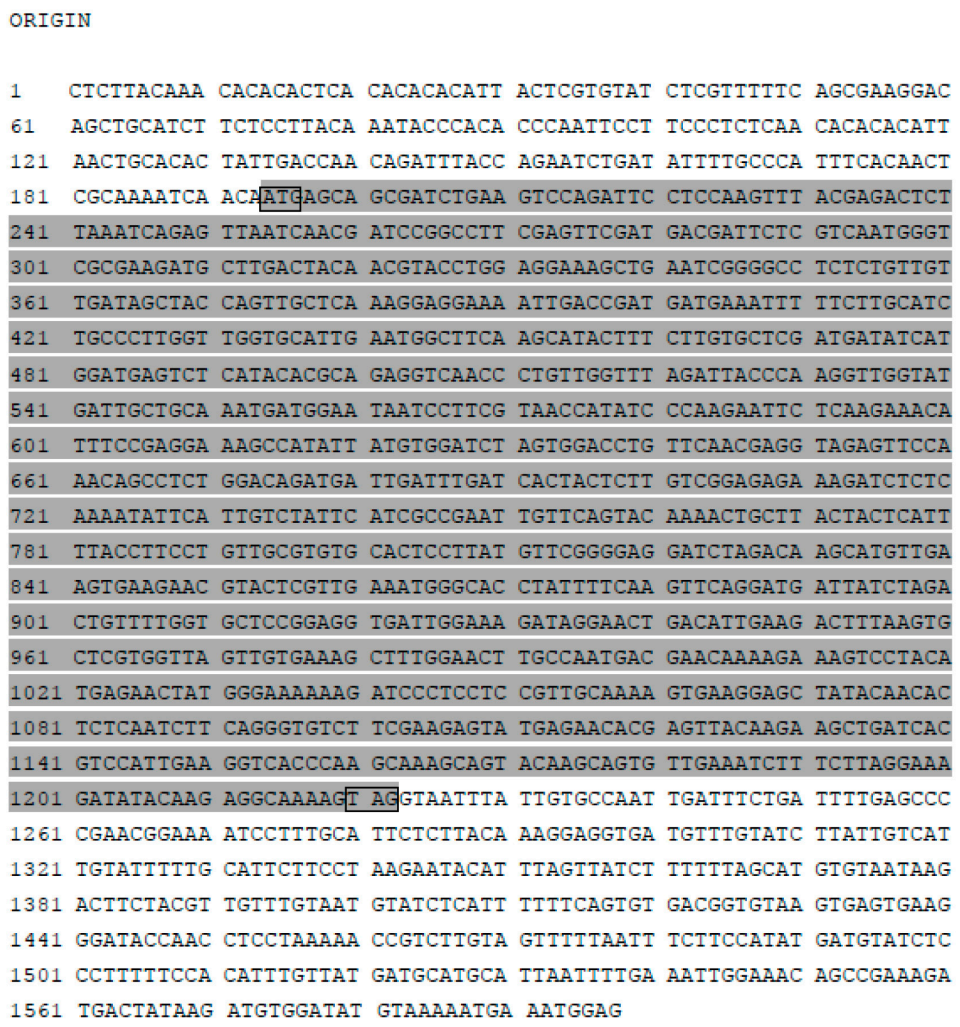

Figure 1. Nucleotide sequence of BbFPS gene from Blumea balsamifera DC. The open reading frame of the sequence is shaded in gray, and the initiation and termination codons are indicated in boxes. 


\section{Amino acid sequence analysis}

Using the ProtParam of the ExPASy program (http://www.expasy.ch/), the B. balsamifera $\mathrm{DC}$ BbFPS protein was analyzed. The results showed that the BbFPS protein contained 342 aa, including 64 negatively charged residues and 41 positively charged residues. The molecular weight was determined to be $39.188 \mathrm{kDa}$. When the BbFPS aa sequence was compared with those of other plants (Arabidopsis lyrata, Aralia elata, Centella asiatica, Citrus x microcarpa, Eleutherococcus senticosus, Glycyrrhiza uralensis, Helianthus annuus, Hevea brasiliensis, and Leibnitzia anandria), no significant differences were found. Alignment of the deduced BbFPS aa sequence with other plant FPS polypeptides showed an identity of approximately $89.07 \%$ between the predicted amino acid sequences derived from the $B$. balsamifera DC BbFPS and other plants (A. lyrata, A. elata, C. asiatica, C. x microcarpa, E. senticosus, $G$. uralensis, H. annuus, H. brasiliensis, and L. anandria) (Figure 2).

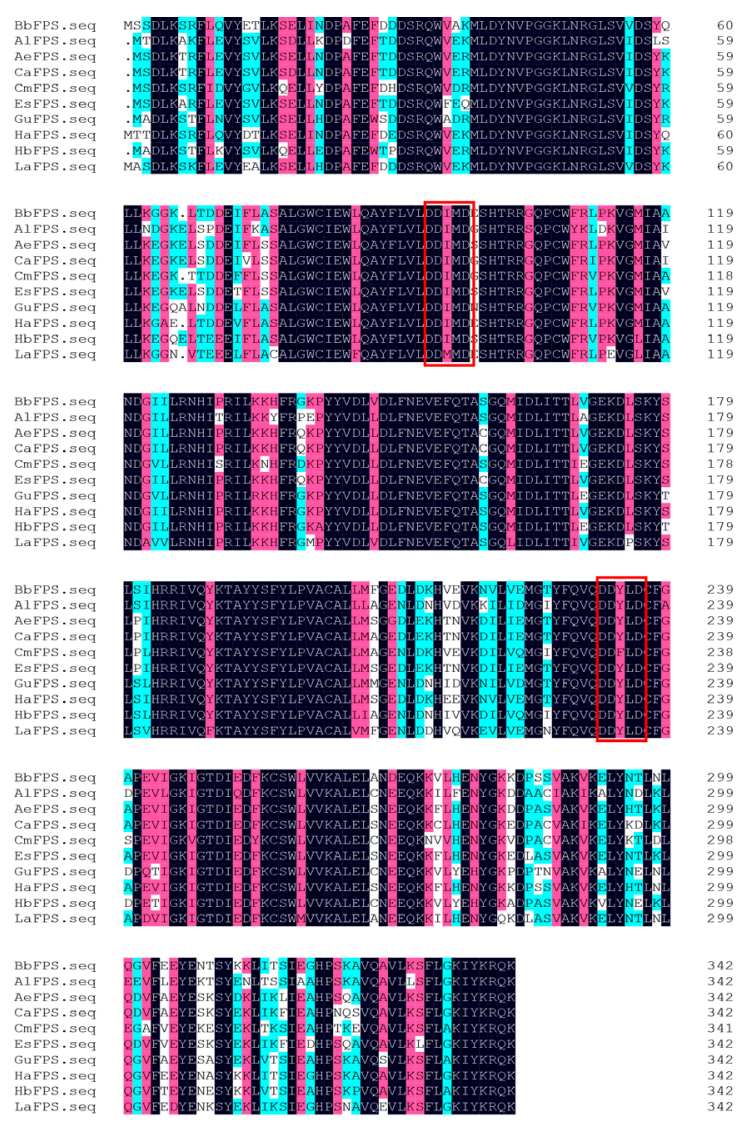

Figure 2. Alignment of predicted amino acid sequences of Blumea balsamifera DC BbTPS and other plant monoTPS. Sequences used for the analysis were obtained from GenBank, the Latin name and accession numbers were as follows: AlFPS (Arabidopsis lyrata XP 002863368.1), AeFPS (Aralia elata ADK12004.1), Ca (Centella asiatica AAV58896.1), CmFPS (Citrus x microcarpa AAK68152.1), AeFPS (Eleutherococcus senticosus AEY77151.1), AeFPS (Glycyrrhiza uralensis ADE18770.1), HaFPS (Helianthus annuus AFW98437.1), HbFPS (Hevea brasiliensis AAM98379.1), and La (Leibnitzia anandria AFW98439.1). DDxxD motifis indicated in redbox. 
The deduced aa sequence of BbFPS contained 2 common terpene synthase family motifs, including DDxxD (aspartate-rich motifs), which is found in nearly all terpene synthases from bacteria and plants and is a putative substrate binding site (Back and Chappell 1995; Bohlmann et al., 1998; Chen, 2007). The DDxxD motif is thought to be a binding site for the diphosphate moieties of the isopentenyl diphosphate and allylic substrates (Ohnuma et al., 1996). Our results are consistent with those of previous studies.

\section{Evolutionary relationship of $B b F P S$ gene}

To determine phylogenetic relationships for the B. balsamifera DC BbFPS protein, the deduced aa sequence was aligned with those of other plant species and a neighbor-joining tree was constructed. Sequences were selected to cover most of the plant species available and to avoid redundancies. As shown in Figure 3, our results revealed that plant FPS proteins were classified into 3 major branches. The phylogenetic tree showed that plants from the same or similar families were clustered into 1 class, such as Achillea asiatica, L. anandria, H. annuus, Matricaria chamomilla, Leucanthemum vulgare, and Chrysanthemum lavandulifolium, which belong to the Asteraceae family. B. balsamifera DC BbFPS is also an Asteraceae plant. This indicated that while phylogenetic analysis of the plant FPS aa did not completely reflect the natural evolution of the relationship between plants, the results might be useful for determining genetic relationships between different plant species (Li and Wang, 2007).

\section{Prediction of protein secondary and 3-D structures}

Prediction of secondary structure of the deduced aa of BbFPS was conducted using the Lasergene software (DNASTAR, Inc., Madison, WI, USA) using the formula described by Garnier et al. (1978). Our results showed that the B. balsamifera DC BbFPS protein contained alpha helices, beta strands, beta-turns, and coil (Figure 4). Among these, alpha helices made up $70 \%$ of the aa sequence.

Analysis of the hydrophilic plot of our aa sequence showed that the hydrophobic areas were mainly in the 3 sections: the $\mathrm{N}$-terminus and $\mathrm{C}$-terminus of the sequence were mainly composed of hydrophilic plots, while in the middle of the BbFPS protein, hydrophilic and hydrophobic aa were alternating. This distribution type of hydrophilic/hydrophobic aa section is closely related to the function of enzyme (Peters et al., 2003; Hyatt et al., 2005, 2007).

To increase the understanding of the 3-D structure of B. balsamifera DC BbFPS genes, homology-based protein models were constructed using the 3-D crystal structure of the farnesyl diphosphate synthase FDS-5, chloroplastic-farnesyl diphosphate synthase 1 chimera with the Swiss-model online service (Benkert et al., 2011) (http://swissmodel.expasy. org/workspace/index.php). The structure of the enzyme was dominated by 29 -helices. The aa of the DDXXD motif (aa indicated with a black dot in Figure 2) was in the area of the enzyme's functional domains, suggesting a functional role for these residues. The QMEAN4 composite score indicated that the prediction model was similar to the actual 3-D structure of BbFPS (Figure 5). The isolation of farnesyl diphosphate synthase gene lays a solid foundation for Blumea balsamifera terpenoid biosynthesis metabolism. 


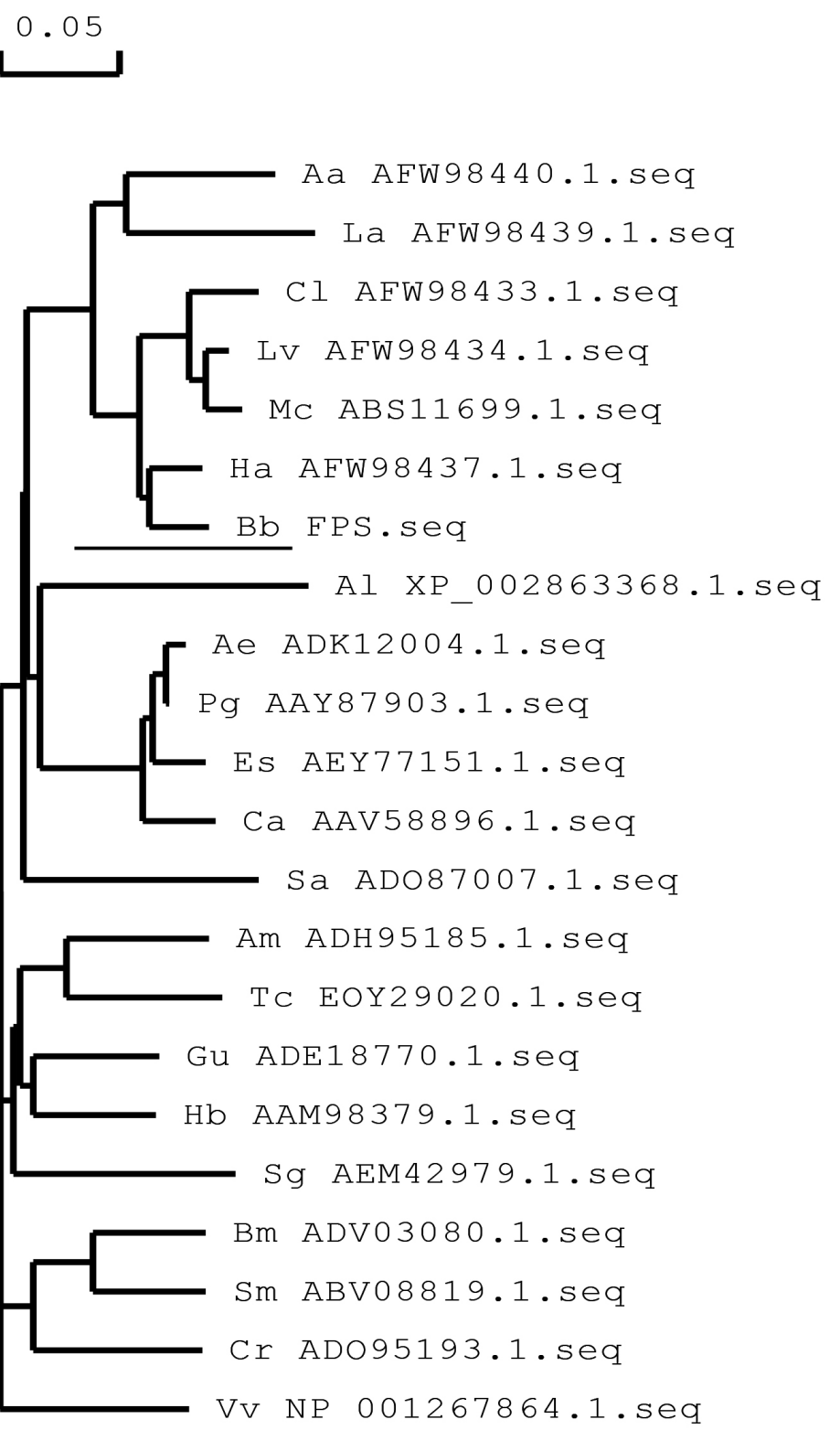

Figure 3. Phylogenetic tree of BbFPS protein from Blumea balsamifera DC and other plant species. Sequences used for the analysis were obtained from GenBank. The Latin name and accession number were as follows: Achillea asiatica (AFW98440.1), Aquilaria microcarpa (ADH95185.1), Arabidopsis lyrata (XP 002863368.1), Aralia elata (ADK12004.1), Bacopa monnieri (ADV03080.1), Catharanthus roseus (ADO95193.1), Centella asiatica (AAV58896.1), Chrysanthemum lavandulifolium (AFW98433.1), Eleutherococcus senticosus (AEY77151.1), Glycyrrhiza uralensis (ADE18770.1), Helianthus annuus (AFW98437.1), Hevea brasiliensis (AAM98379.1), Leibnitzia anandria (AFW98439.1), Leucanthemum vulgare (AFW98434.1), Panax ginseng (AAY87903.1), Matricaria chamomilla (ABS11699.1), Salvia miltiorrhiza (ABV08819.1), Santalum album (ADO87007.1), Siraitia grosvenorii (AEM42979.1), Theobroma cacao (EOY29020.1), and Vitis vinifera (NP 001267864.1). 


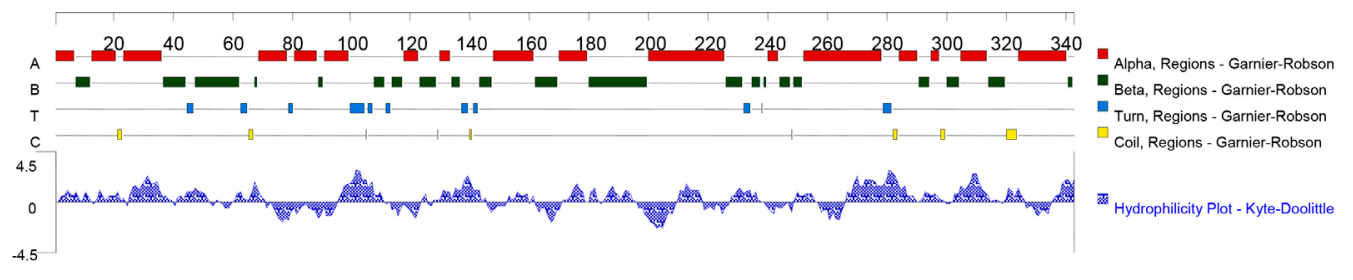

Figure 4. Prediction of secondary structure of BbFPS deduced amino acids sequence; barrel structures are noted above the amino acids sequence. A: alpha helix, B: beta strands, C: beta turns, D: coil.


Figure 5. A. 3-D structure of Blumea balsamifera DCBbFPS, homology-based on the 3-D crystal structure of the farnesyl diphosphate synthase FDS-5, chloroplastic-farnesyl diphosphate synthase 1 chimera. B. QMEAN scores for this model.

\section{ACKNOWLEDGMENTS}

Research supported by the National Nonprofit Institute Research Grant of CATASTCGRI (\#1630032012020) and National Natural Science Foundation of China (\#81202910).

\section{REFERENCES}

Alam MM, Sharmin S, Nabi Z, Mondal SI, et al. H (2010). A putative leucine-rich repeat receptor-like kinase of jute involved in stress response. Plant Mol. Bio. Rep. 28: 394-402.

Ali DMH, Wong KC and Lim PK (2005). Flavonoids from Blumea balsamifera. Fitoterapia 76: 128-130.

Anderson MS, Yarger JG, Burck CL and Poulter CD (1989). Farneyl diphosphate synthase. molecular cloning, sequence, and expression of an essential gene from Saccharomyces cerevisiae. J. Biol. Chem. 264: 19176-19184.

Attucci S, Aitken SM, Gulick PJ and Ibrahim RK (1995). Farnesyl pyrophosphate synthetase from white lupin: molecular cloning, expression, and purification of the expressed protein. Arch. Biochem. Biophys. 321: 493-500.

Back K and Chappell J (1995). Cloning and bacterial expression of a sesquiterpene cyclase from Hyoscyamus muticus and its molecular comparison to related terpene cyclases. J. Biol. Chem. 270: 7375-7381.

Barua NC and Sharma RP (1992). (2R,3R)-7,5'-Dimethoxy-3,5,2'-trihydroxy flavanone from Blumea balsamifera. Phytochem. 31: 4040.

Benkert P, Biasini M and Schwede T (2011). Toward the estimation of the absolute quality of individual protein structure models. Bioinformatics 27: 343-350.

Bohlmann J, Meyer-Gauen G and Croteau R (1998). Plant terpenoid synthases: molecular biology and phylogenetic analysis. Proc. Natl. Acad. Sci. USA 95: 4126-4133. 
Chen CH (2007). Study of anti-tumor effect ofAcanthopanax trifoliatus; understand of knowledge and attitude of Chinese herb between nurses and society in eastern Taiwan. PhD Thesis. Tzu Chi University.Taiwan.

Clarke CF, Tanaka RD, Svenson K, Warmsley M, et al. (1987). Molecular cloning and sequence of a cholesterolrepressible enzyme related to prenyltransferase in the isoprene biosynthetic pathway. Mol. Cell. Biol. 7: 3138-3146.

Delourme D, Lacroute F and Karst F (1994). Cloning of an Arabidopsis thaliana cDNA coding for farnesyl diphosphate synthase by functional complementation in yeast. Plant. Mol. Biol. 26: 1867-1873.

Fazilatun N, Zhari I, Nornisah M and Mas R H (2001). Phytochemical investigation on Blumea balsamifera DC. J. Trop. Med. Plants 2: 17-22.

Fazilatun N, Zhari I, Nornisah M and Mas RH (2004). Free radical-scavenging activity of organic extracts and of pure flavonoids of Blumea balsamifera DC leaves. Food Chem. 88: 243-252.

Garnier J, Osguthorpe D and Robson B (1978). Analysis of the accuracy and implications of simple methods for predicting the secondary structure of globular proteins. J. Mol. Biol. 120: 97.

Hyatt DC and Croteau R (2005). Mutational analysis of a monoterpene synthase reaction: altered catalysis through directed mutagenesis of (-)-pinene synthase from Abies grandis. Arch. Biochem. Biophys. 439: 222-233.

Hyatt DC, Youn B, Zhao Y, Santhamma B, et al. (2007). Structure of limonene synthase, a simple model for terpenoid cyclase catalysis. Proc. Natl. Acad. Sci. USA 104: 5360-5365.

Li R and Wang ZZ (2007). A bioinformatics analyses on farnesyl diphosphate synthase. J. Trop. Subtrop. Bot. 15: 126134.

Norikura T, Kojima-Yuasa A, Shimizu M, Huang X, et al. (2008). Mechanism of growth inhibitory effect of Blumea balsamifera extract in hepatocellular carcinoma. Biosci. Biotechnol. Biochem. 72: 1183-1189.

Ohnuma SI, Hirooka K, Hemmi H, Ishida C, et al. (1996). Conversion of product specificity of archaebacterial geranylgeranyl-diphosphate synthase. Identification of essential amino acid residues for chain length determination of prenyltransferase reaction. J. Biol. Chem. 271: 18831-18837

Peters RJ and Croteau RB (2003). Alternative termination chemistries utilized by monoterpene cyclases: chimeric analysis of bornyl diphosphate, 1,8-cineole, and sabinene synthases. Arch. Biochem. Biophys. 417: 203-211.

Ruangrungsi N, Tappayuthpijaran P, Tantivatana P, Borris RP, et al. (1981). Traditional medicinal plants of Thailand I. Isolation and structure elucidation of two new flavonoids, (2R,3R)-dihydroquercetin-4'-methyl Ether and (2R,3R)dihydroquercetin-4',7-dimethyl Ether from Blumea balsamifera. J. Nat. Prod. 44: 541-545.

Ruangrungsi N, Tantivatana P, Tappayuthpijaran P, Boris RP, et al. (1985). Traditional Medicinal plants of Thailand VI. Isolation of Cryptomeridiol from Blumea balsamifera. J. Sci. Soc. Thailand 11: 47-50.

Saewan N, Koysomboon S and Chantrapromma (2011). Anti-tyrosinase and anti-cancer activities of flavonoids from Blumea balsamifera DC. J. Med. Plant Res. 5: 1018-1025.

Schmidt T, Hillebrand A, Wurbs D, Wahler D, et al. (2010). Molecular cloning and characterization of rubber biosynthetic genes from Taraxacum koksaghyz. Plant Mol. Bio Rep. 28: 227-284.

Sheares BT, White SS, Molowa DT, Chan K, et al. (1989). Cloning analysis and bacterial expression of human farnesyl pyrophosphate synthetase and its regulation in Hep G2 cells. Biochemistry 28: 8129-8135.

Tamura K, Dudley J, Nei M and Kumar S (2007). MEGA4: Molecular evolutionary genetics analysis (MEGA) software version 4.0. Mol. Bio. Evol. 24: 1596-1599. 